\title{
Preliminary simulation research of driver behaviour in response to outdoor advertisements
}

\author{
Tomasz Targosiński ${ }^{1, *}$ \\ ${ }^{1}$ Motor Transport Institute, Warsaw, Poland
}

\begin{abstract}
Advertisements placed near roads can pose a hazard to road safety because they attract the driver's attention. Many attempts to describe and reduce this impact have been done. However, existing regulations treat this problem differently in different countries which demonstrates the significant difficulties in defining and investigating the impact factors of advertisements on driver behaviour. Pilot studies have been done using the eye-tracker and vehicle simulator. Based on the analysis of driver perception in a programmed and repeatable environment an attempt was made to select key factors influencing their attention in terms of the content of advertisements, the size and location in relation to the road and the personality of drivers.
\end{abstract}

\section{Introduction}

The basic intention of outdoor advertising is to attract the observer. From the road safety point of view it is antagonistic to the need of road observation. It is obvious that placing advertisements in the field of view of drivers can negatively influence safety. There are a number of factors that describe and affect distraction during driving. But it is not obvious which ones are really important for safety. They include: the size of billboards, their brightness, position regarding driver field of view. But the content and shape of the ad seem to be most important. Driver perception and behaviour is also influenced by human factors such as habit. The question arises: how to define and measure the influence of ads on drivers' attention. National or local governments decide whether a given advertisement should be allowed or prohibited. As a result, there are different regulations regarding advertisements near roads in different countries, starting from total prohibition of advertisements close to the roads to allowing them nearly everywhere. Nevertheless, there is an objective need to properly regulate this area. A lot of times the decisions to place advertising are subjective [1]. In such attempts there are hidden difficulties with "measuring" the content and shape of the advertisement [2-7]. There were also many experimental tests investigating the influence of ads on driver behaviour. Test were conducted with drivers in real traffic and in driving simulator conditions. They reported various results which depend on arbitrarily chosen test parameters such as lane control, time of reaction, scenario, etc. [8-13], Many tests concern different driving parameters in relation to the very simple state of ads or no ads. The difficulty is that researchers usually try to establish whether the advertisement changes any of measurable parameters such as trajectory, speed or driver workload. The choice of content of

\footnotetext{
* Corresponding author: tomasz.targosinski@its.waw.pl
} 
ads used for the tests is usually subjective with a very general description $[4,8,10]$. Generally, changes were observed in some measurable parameters but other results showed no changes [12]. The influence of the content of an ad was not tested in any of the known research. It defined in a very general and subjective way, e.g. a negative or positive emotional load [13]. The important question is how big is this emotional load, how to classify it and how different persons react depending on personal characteristics and life experiences. When the number of people is relatively low the results might strongly depend on it.

The tests described below were intended to investigate the influence of the content of an ad on driver attention. It was expected that such influence actually occurs but there was no reference as to how much and how. Therefore the goal was to obtain a general view if such a phenomenon existed and to find directions for further research. The tests were not intended to obtain quantitative values. Because of its preliminary character and not many participants, the statistical analysis would not be very useful and might be misleading because of the small number of test persons. The objective was to detect how much the driver can be attracted by different content, size and placement of ad. The investigators look for general results only, using eye tracking which helps to quickly understand whether the driver was attracted or not by an ad. Future more advanced test with restricted test conditions will be the next step and quantitative findings will be studied for their influence on road safety.

\section{The conditions of the experiment}

The tests were conducted in a driving simulator AS1200-6. This simulator consists of a passenger cabin positioned on a system of hydraulic actuators. Before the cabin there is a cylindrical screen for projecting digital dynamic images simulating road scenarios. Additionally, there are displays of rear view mirrors. All vehicle controlling devices and cockpit displays are like those in a regular passenger car. This equipment allows for realistic driving in a computer controlled environment. The main advantage of this kind of experiment was the possibility to create situations which are very difficult to obtain in real road conditions because of safety. Also the repeatability of driving scenarios was higher.

During the experiment, areas of driver sight concentration were registered. For this purpose SMI eye-tracking system was used. Heat maps were used which represent absolute gaze duration. The sensor device was in the form of glasses (Fig. 1.)

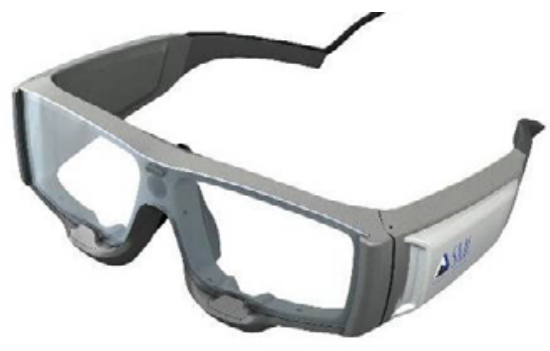

Fig. 1. SMI eye tracking system in the form of glasses.

Driving with the glasses is similar to real conditions. However, some negative phenomena were observed. Some female drivers and people wearing correction glasses were excluded from the experiment because of the sensitivity of the eye tracking device to long eyelashes and optical glasses. Also the calibration of the device can significantly influence the accuracy of the results. For example, if there is a road or traffic sign close to the advertisement, it can be confusing as to what is the object of the driver's interest. If the driver touches the glasses 
during driving, there is a shift and misinterpretations follow. Therefore, a calibration check is crucial to ensure correct interpretation of the results.

Another issue which was considered important for the results was personality traits of the test person. Just as in real road conditions, extremely different reactions were observed starting from no interest for ads for the majority of the subjects to significant interest for two of them. Because of the restricted resources, 19 subjects were chosen from about 100 candidates of different age, sex, and experience as drivers. The results of three of them were not used because of problems with calibration of the eye tracking device and simulator illness. Table 1 shows sex, age and experience as driver.

Table 1. Sex, age and experience of test persons

\begin{tabular}{|c|c|c|c|}
\hline No & Sex & Age & $\begin{array}{c}\text { Experience as } \\
\text { driver }\end{array}$ \\
\hline 1 & $\mathrm{~F}$ & 20 & 2 \\
\hline 2 & $\mathrm{~F}$ & 22 & 1 \\
\hline 3 & $\mathrm{~F}$ & 24 & 6 \\
\hline 4 & $\mathrm{~F}$ & 26 & 8 \\
\hline 5 & $\mathrm{~F}$ & 27 & 8 \\
\hline 6 & $\mathrm{~F}$ & 28 & 10 \\
\hline 7 & $\mathrm{~F}$ & 29 & 10 \\
\hline 8 & $\mathrm{M}$ & 30 & 12 \\
\hline 9 & $\mathrm{~F}$ & 31 & 14 \\
\hline 10 & $\mathrm{M}$ & 31 & 13 \\
\hline 11 & $\mathrm{M}$ & 31 & 13 \\
\hline 12 & $\mathrm{M}$ & 34 & 15 \\
\hline 13 & $\mathrm{M}$ & 37 & 15 \\
\hline 14 & $\mathrm{M}$ & 38 & 20 \\
\hline 15 & $\mathrm{M}$ & 44 & 27 \\
\hline 16 & F & 46 & 30 \\
\hline 17 & $\mathrm{~F}$ & 50 & 33 \\
\hline 18 & $\mathrm{M}$ & 55 & 36 \\
\hline 19 & $\mathrm{M}$ & 55 & 39 \\
\hline
\end{tabular}

The main interest was sensitivity to the contents of the ads. Basically, it was expected that qualitative tendencies will be found because the sample was not big and not versatile enough to formulate general opinions. To attract drivers' attention, the billboards were densely located and differently positioned. Another expectation was to identify the influence of geometric size and position in relation to trajectory of driver sight. Brightness and illumination were not tested because of the restrictions of simulator projectors. To prepare various advertising content, four independent people subjectively selected hundreds of pictures which were rather unusual and not present in real road traffic. All samples were selected by another five different unaware persons with the help of the computer display and eye-tracking system. A notable percentage of chosen advertisements had erotic content, 
because of the general opinion of being eye-catching and often used in outdoor advertisements. The AOI was very clearly defined as ad billboards area introduced in a city and non city driving scenario (see examples on Fig. below).

The whole time the driver's field of view included ads ( 3 to more than 10 big billboards of angular size more than $5^{\circ}$ in the moment of passing). The intention was to attract drivers' attention as much as possible. The test track was about $7 \mathrm{~km}$ long driven for $10-15 \mathrm{~min}$. There were 112 billboards.

Before the tests each participant had about 5 minutes to drive in a similar driving scenario but without advertisement. Drivers were not informed about the purpose of the test. They believed that they were testing eye tracking equipment.

\section{Tests results}

Heat maps were used as a measure of the results representing absolute gaze duration. It is a sufficient measure for preliminary analysis because it represents longer average perception and interest in the object. The value of $0.5 \mathrm{~s}$ was chosen as a minimum to be treated as a noticeable attraction of the driver's attention. Because the environment was specially prepared, a significantly higher number and time of fixations on advertisements was obtained compared to real road conditions. All tested persons have some fixations starting from 2-3 ads during the test drive up to over 30. Most persons look at about 10 ads. Similarly to the real road conditions, there were significant differences between tested drivers. Three of the tested persons (no 3, 10 and 16) had significantly more time and numbers of fixation than the others. It was expected that the reactions will be repeatable (e.g. the same ad and/or positioned in the same place attracts more than one driver). But it was found that it is not such a simple rule. It is interesting that different persons looked at essentially different ads but only some ads were perceived by more than one person. To give an adequate impression nearly all fixation should be presented one by one. But the valuable result of this test is that the selection of subjectively different content causes more interest than ads present in real road conditions. Erotic content was expected to be affecting male drivers. But it attracted also some females even though interest dispersion was observed for different ads among tested drivers. Evaluation of driver interest using general criteria such as erotic content or fashion or unnatural content were found not representative. There is no measure for "intensity" or oddity. To obtain qualitative statistically significant results more test persons would have to participate and/or more test drives would have to be conducted. Otherwise the attributes assigned to content would have the nature of an artefact. The main problem is that drivers have the basic task to control driving trajectory, road sign etc. Time devoted to ads was relatively short. And depending on the traffic situation and driver experience the choice of ad and time was random. In the test the environment was significantly different from that in a commercial ad test with eye-tracking. The time for perception is much longer and other tasks are less important. Nevertheless, a qualitative observation of such a simple measure as heat maps led to a significant conclusion that most of the test persons looked at very many ads which were neither the same nor comparable. Some characteristic examples of perception obtained during tests are presented in Fig. 2. to Fig. 11.

Fig. 2. presents a typical road traffic observation during driving. In Fig. 3. we can see ads competing with the traffic situation and driver behaviour was not risky.

Fig. 4. shows the same place but with a different driver and a longer distraction with another billboard with unusual content (closer to sight trajectory) but without meaningful traffic incentives. 


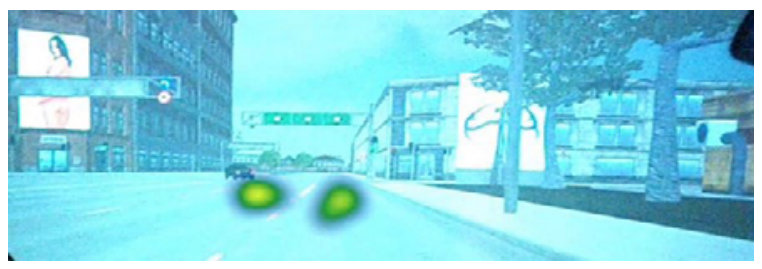

Fig. 2. Looking at the road and oncoming car. (Eye tracking heat map registrations. Yellow colour gaze duration longer than $0.5 \mathrm{~s}$, red colour - longer than $1.0 \mathrm{~s}$ )

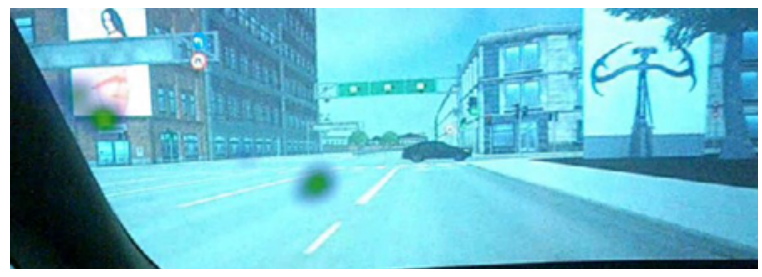

Fig. 3. A moment later. Fixations on ad with erotic content. The same driver as in Fig. 2.

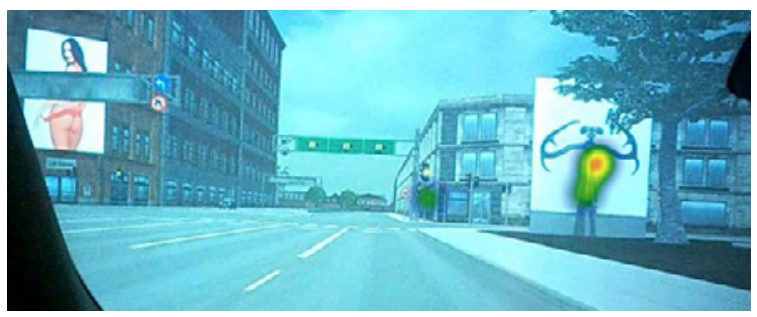

Fig. 4. Another driver. The same place. Longer fixation on unusual picture. Left billboard ignored.

Fig. 5. presents a place where some fixations of different male and female drivers occurred (Registration for the same driver as in Fig. 3.).

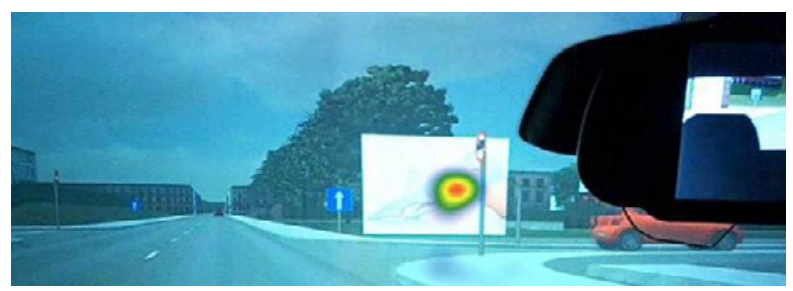

Fig. 5. Fixation on a woman's legs wearing stockings just after the junction. The same driver as in Fig. 3.

It is difficult to define in an objective and repeatable manner the reasons why. The billboard was placed close to the eye position during driving - close to right shoulder. This is because that peripheral view can be used to control driving. It needs more investigation to find if this is content-related or due to the placement in the line of sight, contrast etc. However, other similarly placed billboards caused no fixation of any driver. The same driver ignored many other billboards on route. This means that it is really difficult to find simple rules describing such behaviour because we do not know the driver's thought processes. Another interesting result for the same driver is presented in Fig. 6. In this case the billboard of a relatively big angular size was placed centrally in the driver's field of view. A long perception occurred and the driver tried to read the descriptions on the billboard. This example might be defined as a cognitive riddle but another similar image did not attract attention. The next three examples illustrate a situation with high distraction by erotic content in a demanding traffic 
situation (female driver No.16). There were three similar billboards at the end of a T-shaped junction. In Fig. 7., Fig. 8., and Fig. 9. we can see high distraction when the driver scanned all of the billboards. Finally, a difficult traffic situation occurs (Fig. 9.) with another vehicle crossing perpendicularly.

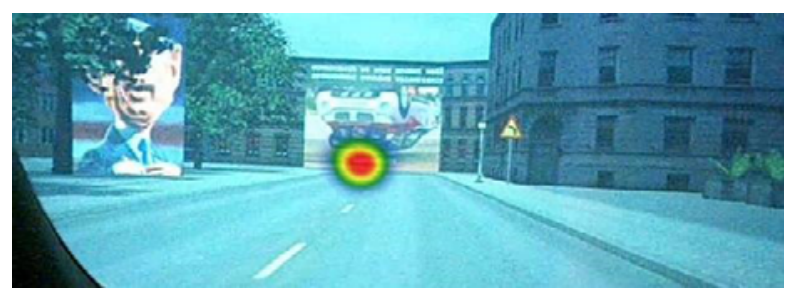

Fig. 6. Look at a strangely looking car (upside down). Reading text. The same driver as above.

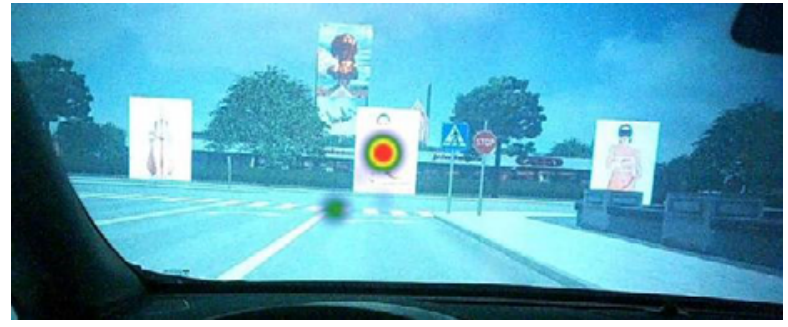

Fig. 7. Long perception of three similar billboards.

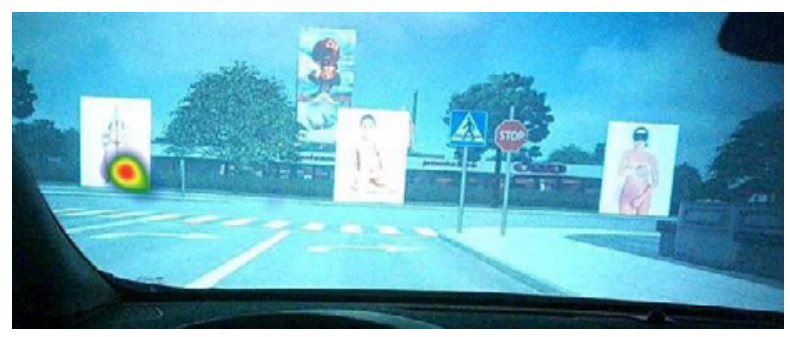

Fig. 8. A while later as above.

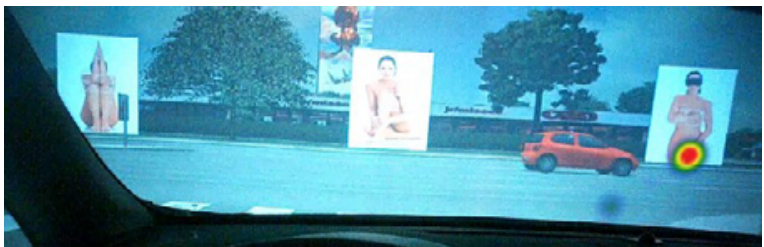

Fig. 9. As above. Perpendicular movement of car.

The examples above clearly show that content and placement of advertisements may influence road safety. Two other drivers looked at this ad (one of three).

A further observed phenomenon was multiplied fixations when the driver approached a billboard (Fig.10., Fig. 11.). 


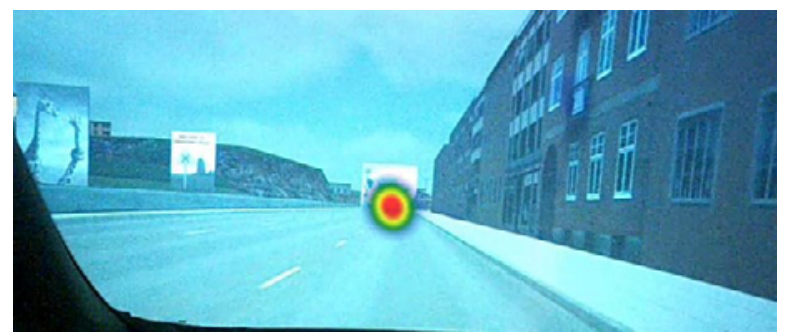

Fig. 10. Fixation on billboard from a distance. Other strange billboards were ignored.

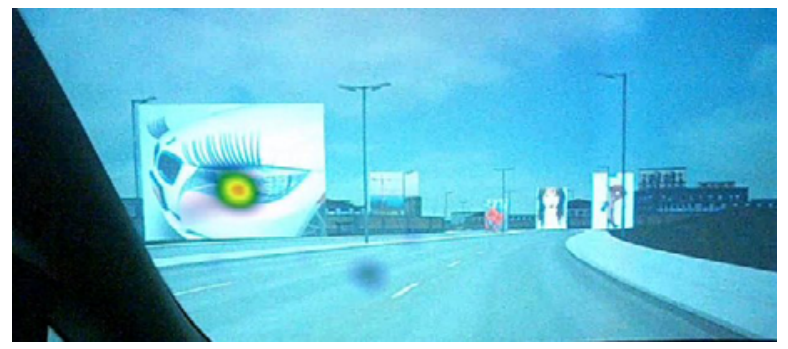

Fig. 11. Multiplied fixations on the same object (Fig. 10.) while ignoring the other.

This situation confirms significant distraction, sometimes caused by trying to read the text, and can be dangerous similarly to the previous example.

\section{Summary}

The tests described above had a preliminary and qualitative nature and their intention was to find the possible ways and reasons why outdoor advertisements influence driver behaviour. During a typical drive there are many factors influencing drivers' perception. Distraction by advertisements depends very strongly on their content but also on sensitivity of a given driver to such particular content. The location of the billboard is also important. It was observed that if the ad is close to the central point of the driver's field of view - and if it is attractive to $\mathrm{him} / \mathrm{her}$, it is watched more often (e.g. Fig. 10.). The big number and high density of unusual billboards presented in the driving simulator environment significantly increase the time of perception in relation to real traffic for the same tested person. To obtain quantitative assessments significantly more tests would have to be conducted with longer times and more test persons. The costs of such tests may be restrictive. It may explain the inconclusive findings of other researchers $[2-6,9,10,13]$. The analysis of the results of above preliminary tests leads to the conclusion that for future tests the drivers should be carefully selected. Attention should be concentrated on drivers most sensitive to ads presence and content. Even if in normal traffic the percentage of such drivers is small, it is likely that they might be more exposed to accidents. The other result of the test is that drivers with more experience (e.g. professional drivers) might be less sensitive to ads as well as young drivers with less experience who are more absorbed with controlling the vehicle and observing the traffic (road signs, another vehicles). A similar situation applies to ad content which seems most important in conjunction with driver personality traits. It is not a simple task to prepare content that will attract all selected "advertisement sensitive" drivers. Nevertheless, the results of the tests show that the need is there and suggest guidelines for future tests. Using unusual ad content seems to be the right decision. The test has also confirmed that erotic content has a relatively more significant effect, however the matter is more complicated and the choice is not obvious. There was a significant dispersion of interests inside the tested group. 


\section{Conclusions}

The highest influence on driver distraction comes from the content of advertising. Other significant factors include personality traits of the tested persons. Drivers were more commonly distracted by billboards close to the centre field of view. The illumination parameters look to be of less importance but need to be tested differently because the driving simulator has a low level of illumination and contrast. Further research is needed which should carefully preselect candidates and ad content. For this purpose carefully chosen personality tests and static computerized tests connected with eye-tracking device will be helpful. Additional factors of driver behaviour should also be measured such as speed changes, trajectory control etc. Driving scenarios can be adapted to different kinds of roads such as city and country roads or motorway. The greatest difficulty is controlling the experiment conditions. The results are highly sensitive to human behaviour, fatigue, training etc.

On the basis of the above tests no quantitative assessment is possible on the impact of ads on traffic safety. But a general observation can be very helpful with preparing future tests with a special focus on ad content. To obtain more adequate results of the influence of ads on road safety, testing conditions must be fixed. As the test showed some people (three of nearly 20) look at a really big number of ads.

Because tests with real drivers are costly and time consuming, a careful preparation of scenarios and selection of test persons is important for reliable findings for a moderate price. There are plans to conduct a more detailed analysis of fixations and saccades of registered data which might give some more information. But at this stage is it clear that it is difficult to find general rules or universal examples of ad content which may be used for all tested persons. Therefore more attention should be paid to a proper choice of test drivers who are "ads sensitive". Such an experiment cannot be repeated too many times because of the effect of habit.

\section{Acknowledgements}

Project "The impact of advertising on the level of traffic safety" is financed by the National Centre for Research and Development (NCBiR) and General Directorate for National Roads and Motorways (GDDKiA), grant agreement DZP/RID-I-33/4/NCBR/2016

\section{References}

1. M.J. Daluge, M. DeLong, L. Hanig, Outdoor Advertising Control Practices in Australia, Europe, and Japan. International Technology Scanning Program Report FHWA-PL-10031 (Federal Highway Administration U.S. Department of Transportation, American Association of State Highway and Transportation Officials, 2011)

2. T. Dukic, C. Ahlstrom, C. Patten, C. Kettwich, K. Kircher, Effects of Electronic Billboards on Driver Distraction, Traffic Inj. Prev. 14(5) pp. 469-476 (2013). doi:10.1080/15389588.2012.731546

3. S.E. Lee, M.J. Mcelheny, R. Gibbons, Driving Performance and Digital Billboards, Prepared for: Foundation for Outdoor Advertising Research and Education (2007)

4. B. Metz, H.-P. Krüger, Do supplementary signs distract the driver?, Transp. Res. Part F Traffic Psychol. Behav. 23 pp. 1-14 (2014). doi:10.1016/j.trf.2013.12.012

5. A. Smiley, B. Persaud, G. Bahar, C. Mollett, C. Lyon, T. Smahel, W. Kelman, Traffic Safety Evaluation of Video Advertising Signs, Transp. Res. Rec. J. Transp. Res. Board. 1937 pp. 105-112 (2005). doi:10.3141/1937-15 
6. B. Wallace, Driver distraction by advertising: genuine risk or urban myth?, in: Proc. Inst. Civ. Eng., (2003)

7. K. Wandachowicz, M. Zalesińska, K. Domke, S. Mroczkowska, P. Skrzypczak, Wielkopowierzchniowe reklamy $\mathrm{z}$ diodami świecącymi a bezpieczeństwo ruchu drogowego, Przegląd Elektrotechniczny. 87 pp. 73-77 (2011)

8. S. Bendak, K. Al-Saleh, The role of roadside advertising signs in distracting drivers, Int. J. Ind. Ergon. 40 pp. 233-236 (2010). doi:10.1016/j.ergon.2009.12.001

9. M.S. Young, J.M. Mahfoud, N.A. Stanton, P.M. Salmon, D.P. Jenkins, G.H. Walker, Conflicts of interest: The implications of roadside advertising for driver attention, Transp. Res. Part F Traffic Psychol. Behav. 12 pp. 381-388 (2009). doi:10.1016/j.trf.2009.05.004

10. D. Kaber, C. Pankok, B. Corbett, W. Ma, J. Hummer, W. Rasdorf, Driver behavior in use of guide and logo signs under distraction and complex roadway conditions, Appl. Ergon. 47 pp. 99-106 (2015). doi:10.1016/j.apergo.2014.09.005

11. S. Milloy, J. Caird, External Driver Distractions:The Effects of Video Billboards and Wind Farms on Driving Performance, in: Handb. Driv. Simul. Eng. Med. Psychol., (CRC Press, 2011). doi:10.1201/b10836-17

12. F. Schieber, K. Limrick, R. McCall, A. Beck, Evaluation of the Visual Demands of Digital Billboards Using a Hybrid Driving Simulator, Proc. Hum. Factors Ergon. Soc. Annu. Meet. 58 pp. 2214-2218 (2014). doi:10.1177/1541931214581465

13. A. Megías, L.L. Di Stasi, A. Maldonado, A. Catena, A. Cándido, Emotion-laden stimuli influence our reactions to traffic lights, Transp. Res. Part F Traffic Psychol. Behav. 22 pp. 96-103 (2014). doi:10.1016/j.trf.2013.09.017 